\title{
Extracting Information from Non Adiabatic Dynamics: Excited Symmetric States of the Bose-Hubbard Model
}

\author{
M. $\operatorname{EACKI}^{a, b}$, D. DELANDE ${ }^{b}$ AND J. ZAKRZEWSKI ${ }^{a, c}$ \\ ${ }^{a}$ Instytut Fizyki Mariana Smoluchowskiego, Uniwersytet Jagielloński, W.S. Reymonta 4, 30-059 Kraków, Poland \\ ${ }^{b}$ Laboratoire Kastler-Brossel, UPMC, ENS, CNRS, 4 Place Jussieu, F-75005 Paris, France \\ ${ }^{c}$ Mark Kac Complex Systems Research Center, Uniwersytet Jagielloński, W.S. Reymonta 4, 30-059 Kraków, Poland \\ Using Fourier transform on a time series generated by unitary evolution, we extract many-body eigenstates \\ of the Bose-Hubbard model corresponding to low energy excitations, which are generated when the insulator- \\ superfluid phase transition is realised in a typical experiment. The analysis is conducted in a symmetric external \\ potential both without and with a disorder. A simple classification of excitations in the absence disorder is \\ provided. The evolution is performed assuming the presence of the parity symmetry in the system rendering \\ many-body quantum states either symmetric or antisymmetric. Using symmetry-breaking technique, those states \\ are decomposed into elementary one-particle processes.
}

PACS: $67.85 . \mathrm{Hj}, 03.75 . \mathrm{Kk}, 03.75 . \mathrm{Lm}$

\section{Introduction}

A gas of ultracold atoms in a sufficiently deep optical lattice is well described by a tight-binding model the so-called Bose-Hubbard (BH) model as suggested by Jaksch and Zoller in their seminal article [1]. The authors predicted the occurrence of a Mott insulator (MI) to superfluid (SF) phase transition, later realized experimentally [2]. Let us note parenthetically that the notion of "Bose-Hubbard model" may be considered an example of validity of the zeroth theorem of the history of science [3], as it should rather be called the GerschKnollman model [4].

Optical lattices provide a superb experimental possibility not only by enabling to implement the BH Hamiltonian but also providing means to control the parameters of the model. Although variations of the lattice depth modify both the on-site interaction and the tunneling rate between sites, there is an independent method of manipulating the strength of the interaction, by tuning the scattering length of atoms using magnetic Feshbach resonances [5]. Optical and microwave Feshbach resonances have also been developed $[6,7]$.

Random on-site disorder within the Bose-Hubbard Hamiltonian causes the existence of a new insulating, yet gapless phase - the Bose glass [8]. This result has been generalized to the pseudodisorder realized by a bichromatic optical potential [9]. Similarities and differences between effects due to these two types of disorder are discussed in $[10,11]$.

To study experimentally the Bose glass phase [9], an ultracold atomic gas is first prepared in a trap. The optical lattice is then switched on, driving the system through the insulator-superfluid phase transition in a finite time. When a phase transition is classical, such a quench may be described by the Kibble-Zurek mechanism [12, 13], leading to the presence of several excitations in the final phase. For quantum phase transitions, the situation was found to be similar in an array of the Josephson junctions [14]. Investigation of quantum Ising models [15] and homogeneous BH model [16] shows that the number of defects scales algebraically with the quench time.

In our previous research [17], we showed that experimental setup (approximated within the BH model) used in [9] for the realization of the ground state (a series of the Mott insulators without disorder, a Bose glass with strong disorder) leads to a significant (90\% without disorder, $>99.999 \%$ with the strong disorder) depletion of the ground state, making the interpretation of this wave packet as a Bose glass less obvious.

In this article, we continue our theoretical analysis by extracting eigenstates of the Bose-Hubbard Hamiltonian excited by quenching and comparing them to the ground state and the dynamically created wave packet. We focus on the special situation when the external potential possesses parity symmetry and the realization of the pseudo-disorder (bichromatic field) respects that symmetry.

\section{The method of analysis}

As in [17] we take as an example the experiment of the Florence group (for details see the original work [9]). An harmonic trap was used to confine the cold atomic gas; then a two-dimensional optical lattice potential (the "transverse" lattice) is ramped up to create a two-dimensional array of independent (if the lattice is sufficiently strong, tunneling between tubes is inhibited) one-dimensional tubes. The same ramp is used to switch on the optical potential along the tubes. The latter potential may either be a pure "optical lattice" or a bichromatic lattice realizing a pseudo-random disorder.

The recoil energy $E_{\mathrm{R}}=h^{2} /\left(2 M \lambda^{2}\right)$ is used as the energy scale, $\lambda=830 \mathrm{~nm}$ being the wavelength of laser beams forming the optical lattices (both transverse and along the tube), and $M$ - the mass of an atom. Initially, the only external potential present is the harmonic trap. 
Then the 2D optical lattice creating tubes, optical lattice potential along tubes and an additional much weaker optical lattice creating disorder are ramped together (exponential ramping lasts $100 \mathrm{~ms}$ in total). The additional optical lattice is created with a different laser, with wavelength $\lambda_{\mathrm{d}}=1076 \mathrm{~nm}$.

The transverse optical lattice potential maximal height is $s_{\perp}=35$ (in recoil energy units), the maximal height of the lattice along the tubes $-s=14$. Notice that we follow [17] in rescaling the experimental $s$ parameters by a $7 / 8$ factor, for a discussion see [17]. As it is much higher than the lattice potential along the tubes, the transverse lattice makes the system an independent union of $1 \mathrm{D}$ systems at the early stages of the ramp. We model a single tube using the 1D Bose-Hubbard Hamiltonian (tight-binding approximation of a full, second quantized Hamiltonian):

$$
\begin{aligned}
\hat{H} & =-J \sum_{j=0}^{N-1}\left(\hat{b}_{j}^{\dagger} \hat{b}_{j+1}+\hat{b}_{j+1}^{\dagger} \hat{b}_{j}\right) \\
& +\frac{U}{2} \sum_{j}^{N} \hat{n}_{j}\left(\hat{n}_{j}-1\right)+\sum_{j}^{N} \epsilon_{j} \hat{n}_{j},
\end{aligned}
$$

where $\hat{b}_{j}$ is an annihilation operator of a particle at the $j$-th site and $\hat{n}_{j}=\hat{b}_{j}^{\dagger} \hat{b}_{j}$ - the corresponding particle number operator. Both $J$ and $U$ depend on parameters $s$ and $s_{\perp}[1]$. The lattice depth $s$ increases in time, making $U$ (slowly) increasing and $J$ (exponentially) decreasing. The underlying assumption within the $\mathrm{BH}$ Hamiltonian is that the Hilbert space is restricted only to the lowest Bloch band of the lattice. We perform the evolution only for lattices deep enough to justify neglecting higher bands. The $\epsilon_{j}$ represents the energy offset of the on-site energy at site $j$ :

$$
\epsilon_{j}=\frac{1}{2} M \omega^{2} a^{2}\left(j-j_{0}\right)^{2}+s_{\mathrm{d}} E_{\mathrm{R}} \sin ^{2}\left(\frac{\pi j \lambda}{\lambda_{\mathrm{d}}}\right) .
$$

The first term comes from the external harmonic trap potential ( $\omega$ is the trapping frequency, $j_{0}$, not necessarily an integer, is the center of the trap), the second one corresponds to the additional optical potential introducing the disorder created by laser with wavelength $\lambda_{\mathrm{d}}$. The parameter $s_{\mathrm{d}} \ll s$ is its amplitude (in recoil energy units) - when $s_{\mathrm{d}}>1$ it is considered strong $[9,10]$. As in [17], we use the $\mathrm{BH}$ model only for $s>4$, where it is applicable. We assume that initially the gas is in the ground state in a superfluid state for $s=4$, and that the hopping through the transverse lattice is negligible.

Matrix product states $[18,19]$ are used to represent the states and the time evolving block decimation [20] (essentially equivalent to time-dependent density matrix renormalization group method [21]) algorithm is used for the time evolution during which $s$ is increased exponentially as $s(t)=A(\exp (t / \tau)-1)$. The exponential ramp is characterized by $\tau=30 \mathrm{~ms}$. At the end of the ramp which is not adiabatic - we have created a wave packet, a linear combination of the ground state and various ex- cited states. To characterize the properties of this dynamically created wave packet, it is important to know the properties of the significantly populated eigenstates. To reach this goal, when the final $s$ value is reached, we further continue to evolve the wave packet using the time-independent Hamiltonian with constant $s$. Let us denote by $\left|e_{i}\right\rangle$ the eigenbasis of the final Hamiltonian. The evolution of the wave packet under constant $s$ is given by

$$
|\psi(t)\rangle=\sum_{i} \exp \left(-\frac{\mathrm{i}}{\hbar} E_{i} t\right) c_{i}\left|e_{i}\right\rangle,
$$

and the Fourier transform (FT) of the autocorrelation function

$$
C(t)=\langle\psi(0) \mid \psi(t)\rangle=\sum_{i}\left|c_{i}\right|^{2} \exp \left(-\mathrm{i} \frac{E_{i} t}{\hbar}\right)
$$

yields $E_{i}$ 's - the eigenenergies of the final Hamiltonian and the overlaps $\left|c_{i}\right|^{2}$ as discussed in [17]. The extraction of the ground state using imaginary time propagation was also performed. Here, we extend this analysis by extracting from the dynamics also the excited eigenstates with large overlap - those contribute most significantly to the dynamical wave packet and thus provide an understanding of the character of that wave function.

We focus on the situation where parity symmetry is present making the eigenstates either symmetric or antisymmetric. This makes it possible to apply a symmetry-breaking analysis of the final state.

To find a given $\left|e_{i}\right\rangle$ we perform a Fourier transform of the various $|\psi(t)\rangle$ (each represented by a matrix product state, MPS) on a discretized sample, $t=n \delta t$, with $n$ an integer. This requires a method for adding many-body states within the MPS state representation; details concerning the method including details on its validity will be published elsewhere.

\subsection{Bose-Hubbard model in the absence of disorder}

Consider first the example of $s_{\mathrm{d}}=0$, i.e. a pure BoseHubbard model. Figure 1 shows the FT of the autocorrelation function of the wave packet which was obtained using an exponential ramp. As discussed before [17], the wave packet has about $10 \%$ (squared) overlap with the ground state (the peak at zero relative energy in Fig. 1, corresponding to energy $E \approx 119.188$ ) with 4 other states contributing with higher or similar overlap. Using the procedure sketched above we extract the eigenstates corresponding to the dominant contributions.

Let us take a look at these states in some detail. In Fig. 2, we show the average occupation numbers $\left\langle n_{j}\right\rangle$ of sites $j$ for the ground state $(\mathrm{G})$ as well as states P1 and $\mathrm{P} 2$ corresponding to peaks bearing the same name in Fig. 1. Observe that occupations of all three states coincide within broad steps of the Mott plateau (with integer occupation of sites). For the ground state, the central $\langle n\rangle=3$ zone is broadest, for the two excited states, one particle from the $\langle n\rangle=3$ zone is transferred either to the left or to the right. Since we consider a symmetric potential problem (the center of the harmonic trap coincides with the site $j=41$ ), the eigenstates are either 


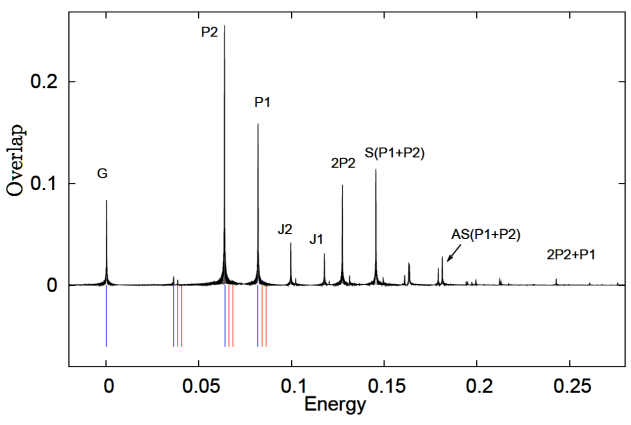

Fig. 1. Fourier transform of the autocorrelation function, Eq. (4), obtained dynamically for $s=14$ after switching on the lattice without the secondary lattice, i.e. for $s_{\mathrm{d}}=0$ (no disorder). The experimental [9] exponential ramp of $100 \mathrm{~ms}$ is used, all other parameters are taken as closely as possible to the experimental situation, with $N=151$ particles on $M=81$ sites. The peaks appear at energy levels of the system (measured with respect to its ground state), with an intensity equal to the squared overlap with the wave packet. About ten states are significantly excited, proving that the preparation is not adiabatic in a strict quantum mechanical sense. The stick spectrum shown in a mirror is the prediction for the energies using a simple separable ansatz allowing the identification listed in the figure - see text for discussion. The $\mathrm{S}(\mathrm{P} 1+\mathrm{P} 2)$ corresponds to a symmetric combination of both $\mathrm{P} 1$ and $\mathrm{P} 2$ excitations, the $\mathrm{AS}(\mathrm{P} 1+\mathrm{P} 2)$ to an asymmetric combination.

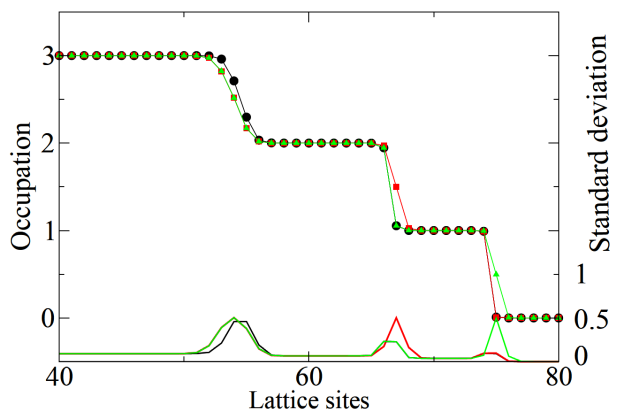

Fig. 2. Occupation of sites (left vertical axis) $\left\langle n_{j}\right\rangle$ for the ground state (black circles connected by a line) and two excited states $\left|\psi_{2}\right\rangle$ (red squares) - corresponding to the peak P2 in Fig. 1 and $\left|\psi_{1}\right\rangle$ (green triangles, peak P1) which are significantly populated during the turn-on of the lattice. Due to the symmetry of the problem, half of the system is shown only. Right vertical axis shows standard deviation of the occupation number $\Delta_{j}=\sqrt{\left\langle n_{j}^{2}\right\rangle-\left\langle n_{j}\right\rangle^{2}}$. Low values correspond to an insulating Mott state, excitations occurring in the SF zones lead to an increase of the standard deviation.

symmetric or antisymmetric with respect to the trap center. This symmetry is not broken when parameters of the Hamiltonian are varied during switching on of the lattice, therefore only symmetric eigenstates are populated. This explains half integer occupations on the border between $\langle n\rangle=2$ and $\langle n\rangle=1$ zones for $\mathrm{P} 2$ or $\langle n\rangle=1$ and $\langle n\rangle=0$ zones for $\mathrm{P} 1$.

It seems, therefore, that basic excitations in the system correspond to transfer of particles between edges of the Mott zones. This is because the SF regions between the zones are very small $(s=14$ corresponds to $J / U=0.0133$, very deep in the Mott regime). If this is the case, can the most significant excitations be explained by such transfers?

To test this hypothesis, let us consider first the system at $J=0$.

\subsubsection{Ground and excited states for $J=0$}

For $J=0$, the ground state is well known. All eigenstates, in particular the ground state, are product of the Fock states at different sites - at each site $i$ we put exactly $n_{i}$ particles

$$
|\psi\rangle=\bigotimes_{i=0}^{M}\left|n_{i}\right\rangle .
$$

To create the ground state with $N$ particles, one has to perform $N$ times the following procedure: find the site whose local energy (energy taking into account the harmonic trap (2) together with interaction with particles already present on the site) is the least and put the particle into that site. Without disorder, the ground state has a well-known wedding cake form - see Fig. 3 .

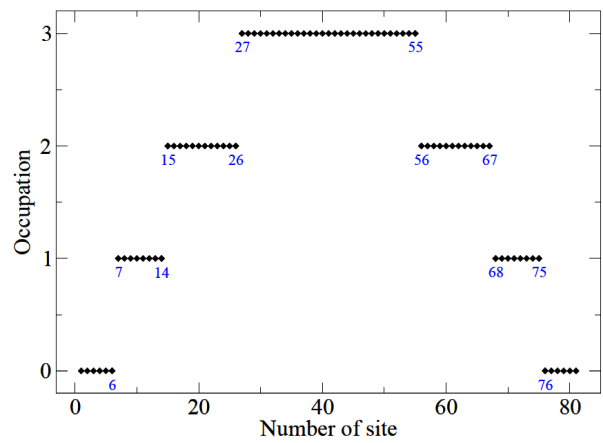

Fig. 3. Ground state of the BH model for $J=0$, composed of a series of insulating Mott plateaus with the familiar wedding cake shape. In blue, sites at the edge of the Mott zones.

To get the low-lying excited state, one should take a few particles (perhaps one) and move it to a different place. To achieve low energy difference, the particle must be put back in such a way that the resulting particle distribution is close to optimal. Some possibilities are shown in Fig. 4.

Consider now our exemplary case of $N=151$ particles on 81 sites. The ground state at $J=0$ is shown in Fig. 3 . At site numbers marked in the figure, there are sudden changes of local occupation numbers defining the edges of different occupation zones (the Mott plateau).

As the ground state is $\mathbb{Z}_{2}$-symmetric (i.e. even with respect to reflection at the center of the trap), several $\mathbb{Z}_{2}$-symmetric one-particle excitations may be generated by taking one particle from one of sites 7,15 or 27 and 


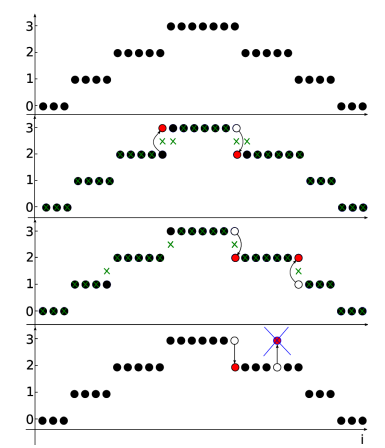

Fig. 4. Ground state of the Hamiltonian for $J=0$ (a). For a better visualization, the number of sites is reduced from $M=81$ in simulations of the experiment to $M=31$. Particle removed from a certain MI zone may be placed in the other zone (b) or put back at the same zone, but on the other side from the center of the trap effectively shifting the zone to one side (c). After symmetrization of the state, a distribution denoted by green crosses will be obtained. Bottom plot (d) shows an example of other possibility that exists for $J=0$. For $J>0$, this type of excitation is unlikely to be observed in a real experiment, due to its dynamical instability and a too high excitation energy.

moving it to one of sites 6,14 or 26 . Symmetric possibilities are when the particle is taken on the right side (sites $75,67,55$ symmetric partners of respectively $7,15,27$ ) and/or is moved to sites $76,68,56$ on the right side. This generates 9 different energy combinations that are listed in Table, each combination being 4 times degenerate (left/right for the particle removed, left/right for the particle added).

\subsubsection{Nonzero small $J$, no disorder}

Let us come back to the "physical" case of small $J / U=$ 0.0133 corresponding to the lattice with height $s=14$ and the FT of the correlation function of dynamically obtained wave packet shown in Fig. 1. The ground state energy $E=119.185$ is quite close to $J=0$ estimate (see Table) - the difference is on the fourth significant digit. Still this difference is much larger than the excess energy of excitations listed in Table - it is mainly due to the important role of tunneling in the narrow SF strips. The exemplary extracted states, see Fig. 2, suggest that the excitations are due to transfers between edges of the Mott zones. Therefore, the "excess energy" of such excitations above the ground state can be approximated by the $J=0$ energy excess (remember we consider deep optical lattice - this argument might not hold close to the SF-MI transition). By inspection of the excess energies, we can then identify several important contributions to the wave packet in terms of elementary excitations and their multiplicities! The classification is included in Fig. 1.

It is apparent that the main origin of the nonadiabaticity comes from P1 and P2 excitations and their multiplicities. The corresponding elementary particle exchange
TABLE

Comparison of 9 elementary excitations for $J=0$. Each one particle excitation corresponds to moving a particle from site $i$ to site $j$ (due to symmetry with respect to site 41, only excitations where a particle is moved from the left side to the right side are shown). Third column gives the energy of that excitation. Fourth column gives the excess energy with respect to the ground state. Fifth column identifies the state with state obtained for small $J$ in Fig. 1. Last column give the degeneracy of excitation for $J=0$, with, inside the parentheses, the degeneracy expected in the low $J$ limit.

\begin{tabular}{c|c|c|c|c|c}
\hline \hline$i$ & $j$ & Energy & $\begin{array}{c}\text { Excess } \\
\text { energy }\end{array}$ & Notation & Degeneracy \\
\hline- & - & 119.332 & 0 & $\mathrm{G}$ & 1 \\
27 & 56 & 119.368 & 0.036 & $\mathrm{~S} 3$ & $4(2)$ \\
15 & 56 & 119.370 & 0.038 & $\mathrm{~T} 2$ & 4 \\
7 & 56 & 119.373 & 0.041 & $\mathrm{~T} 1$ & 4 \\
27 & 68 & 119.396 & 0.064 & $\mathrm{P} 2$ & 4 \\
15 & 68 & 119.398 & 0.066 & $\mathrm{~S} 2$ & $4(2)$ \\
7 & 68 & 119.401 & 0.069 & $\mathrm{D} 1$ & 4 \\
27 & 76 & 119.414 & 0.082 & $\mathrm{P} 1$ & 4 \\
15 & 76 & 119.416 & 0.084 & $\mathrm{D} 2$ & 4 \\
7 & 76 & 119.418 & 0.086 & $\mathrm{~S} 1$ & $4(2)$
\end{tabular}

process is a loss of particle from the highest $\langle n\rangle=3$ Mott zone to $\langle n\rangle=2$ (P2) or to $\langle n\rangle=1$ (P1).

Excitations of type $\mathrm{S}$ are slightly different: indeed, there, the particle is removed from the Mott plateau $n$ and added in the Mott plateau $n-1$. When the two sites are on opposite sides, it simply corresponds to a right or left shift of plateau $n$ by one site. For example, the S3 component has a squared overlap of less than $1 \%$, still the analysis of the associated state by our method confirms that assignment. This process for other Mott zones (S2 and S1) is not observed. There is, however, another possibility for $\mathrm{S}$ excitations: if the $i$ and $j$ neighboring sites are on the same side, it is associated with a hole in the $n$-plateau (can be viewed also as an extra particle in the $n-1$ plateau), and - because $i$ and $j$ are neighbors (with slightly different energy offset at each side) it costs some kinetic energy. Thus one can expect the 4 -fold degeneracy of the $\mathrm{S}$ excitations at $J=0$ to be rapidly lifted to a 2-fold degeneracy only for relatively small $J$. Also, the same-side excitation is dynamically unstable and unlikely to be significantly excited in our wave packet.

We do not observe significant contributions of processes involving exchange of particles between $\langle n\rangle=1$ to $\langle n\rangle=2$ zones only (D type). A qualitative explanation for that fact might be that formation of $\langle n\rangle=1$ and $\langle n\rangle=2$ zones takes time earlier in the ramping-up process (for larger $J / U$ ). Then tunneling more efficiently redistributes particles between sites, also the changes of $J$ and $U$ occur more slowly due to an exponential shape of the ramp. Moreover, observe that excitations when the 
particle is promoted to the highest $\langle n\rangle=3$ level at the expense of shrinking the lower Mott zone (T1 and T2) are not observed. This seems physically understood quite naturally. With the increase of the lattice depth the highest Mott zone also increases at consecutive avoided crossings if passed adiabatically. If it does not have time to increase sufficiently, P1 or P2 excitations are created. But no avoided crossings of $\mathrm{T} 1$ or $\mathrm{T} 2$ process may occur.

With that in mind, we can actually identify the dominant processes contributing to the $\mathrm{P} 1$ and $\mathrm{P} 2$ excitations. Consider P1 only (for P2 the same arguments apply except different lower Mott zones are involved). Recall that P1 corresponds to loss of one particle at the edge of the $\langle n\rangle=3$ zone, say on the left side (site $i=27$ ) with the additional particle appearing at the edge between the $\langle n\rangle=0$ and $\langle n\rangle=1$ zones. This may occur at the same side of the wedding cake (site $j=6$ in our example) or on the opposite side of the center (at site $j=76$ ). A more detailed analysis is given in the next section.

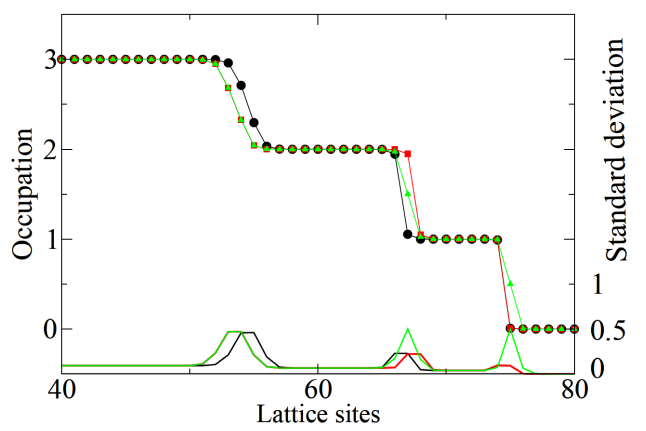

Fig. 5. Exemplary excitations involving transfer of two atoms from the $\langle n\rangle=3$ zone, either both to $\langle n\rangle=2$ (one on the left, one on the right) - 2P2 excitation, red squares connected by line; or one to $\langle n\rangle=2$ and the other one to $\langle n\rangle=1-P 1+P 2$ (green triangles). Black circles give the ground state for reference. Due to the symmetry of the potential only half of the trap is shown. The right vertical axis shows standard deviation of the occupation number.

Similarly, we may easily understand the $\mathrm{P} 1+\mathrm{P} 2$ or $2 \mathrm{P} 2$ processes (compare Fig. 5) contributing significantly to the FT of the autocorrelation function shown in Fig. 1. Then, the Mott $\langle n\rangle=3$ zone loses one particle at each side, both particles moving to lower zones. The $2 \mathrm{P} 2$ case is symmetric, on both sides a particle appears at the border between $\langle n\rangle=1$ and $\langle n\rangle=2$ zones.

For $\mathrm{P} 1+\mathrm{P} 2$ a symmetric combination is created, $\mathrm{P} 1$ on the left and $\mathrm{P} 2$ on the right or vice versa. The classification denoted in the correlation function plot have been confirmed by extracting the excited states responsible for those peaks.

Apart from simple excitations, easily identifiable in the $J=0$ limit, there are other excitations involving particles in narrow superfluid strips separating different Mott zones. Two of these excitations, denoted as J1 and J2 in Fig. 1 are shown in Fig. 6.

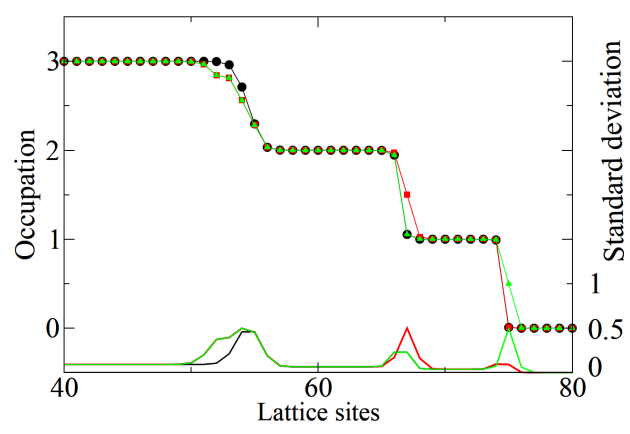

Fig. 6. Examples of excitations involving particles in superfluid zones. Excitations denoted as J2 and J1 in Fig. 1 are shown as red squares and green triangles, respectively and compared with the ground state (black circles). The excitation leads to broadening of the SF zone separating the $\langle n\rangle=3$ and $\langle n\rangle=2$ Mott zones. Due to the symmetry only half of the trap is shown. The right vertical axis shows standard deviation of the occupation number. The J2 excitation corresponds to the transfer of one particle from a SF zone to a $\langle n\rangle=2$ Mott plateau, J1 to the $\langle n\rangle=1$ zone as could be traced back from the standard deviation plot. The standard deviation of the occupation number (right scale) confirms the broadening of the SF region between the $\langle n\rangle=3$ and $\langle n\rangle=2$ Mott zones.

\section{Parity symmetry breaking}

It is possible to describe the state $\mathrm{P} 1$ (this reasoning is not limited to this state, the presented method is rather general) further by describing correlations within eigenstates of $\hat{H}$. As shown in Sect. 2.1.1, in the limit $J \rightarrow 0$, the $\mathrm{P} 1$ excited state has a 4-fold degeneracy, with 2 states symmetric by parity symmetry and 2 antisymmetric ones. When both the Hamiltonian and the dynamically excited wave packet is symmetric, the overlaps with antisymmetric states vanishes, making them invisible in the FT of the autocorrelation function. The two remaining (symmetric) states are strictly degenerate only for $J=0$. For non-zero $J$, they are coupled via tunneling of one particle from one side of the $\langle n\rangle=3$ plateau to the other side. The associated amplitude is very small, and the two states are almost degenerate, that is not resolved in out FT over a finite time interval.

However, if we now break the symmetry parity and propagate the same wave packet with a slightly asymmetric Hamiltonian - for example obtained by shifting the trap center by $\delta \ll 1$ with respect to the lattice the 4-fold degeneracy as well as the selection rules forbidding the excitation of the antisymmetric states will be broken, and one expects to observe the $\mathrm{P} 1$ peak to split in a multiplet of 4 peaks.

The FT of the autocorrelation function in Fig. 7 clearly shows these 4 components. As expected the height of the $\mathrm{P} 1$ peak for $\delta=0-0.158$ - is now shared by the four components of the multiplet, the sum of heights being 0.154 .

For $J=0$, each of constituents of the P1 multiplet differs from the ground state by a particle jump from one of sites 27 and 55 to one of sites 6 and 76 . These 


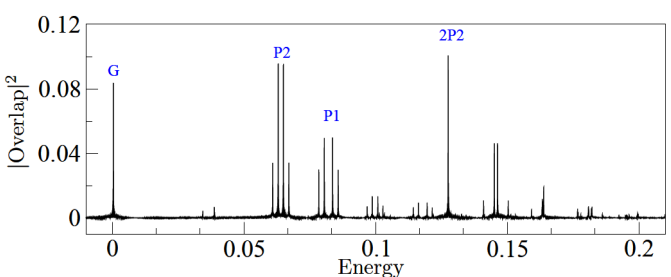

Fig. 7. Fourier transform of the autocorrelation of a wave packet evolved for $\delta=0.03$. One clearly observes splitting of eigenenergy peaks with a multiplicity corresponding to the degeneracy at $\delta=0$ given in Table. Labels associate the 4 -fold group of peaks for the symmetry broken case to a corresponding single peak at $\delta=0$. Let us note that the $2 \mathrm{P} 2$ peak does not split, as it corresponds to a non-degenerate excitation.

particle jumps break the parity symmetry and make the energies dependent on $\delta$ in the first power. Outer peaks correspond to jumping particles over a long distance 55 to 6 or 27 to 76 , inner, higher peaks correspond to one-sided jumps: 27 to 6 and 55 to 76 . It turns out that values $E_{0}^{i}-E_{\delta}^{i}$ calculated for $J=0$ are very close to those extracted from Fig. 7.

It might have been tempting to argue that the larger the distance between two sites $i$ and $j$ the lesser the probability of finding an excitation that differs from the ground state by hopping a particle from site $j$ to site $i$. The heights of peaks that are results of splitting a symmetric ground state show a different picture. The probability is certainly lower, but the order of magnitude remains largely the same - the difference is by a factor of 1.5-4 depending on the symmetric eigenstate being considered.

The P2 peak behaves exactly like the $\mathrm{P} 1$ peak with a quadruplet appearing when parity is broken, see Fig. 7 . The $2 \mathrm{P} 2$ peak behaves very differently, with a single peak surviving keeping all the weight. As noticed above, the $2 \mathrm{P} 2$ case corresponds to 2 atoms jumping from the $\langle n\rangle=$ 3 to each of the $\langle n\rangle=2$, a symmetric non-degenerate state, like the ground state, in the $J=0$ limit.

\section{The disordered BH model}

The presence of the on-site disorder, e.g. in the form (2) for $s_{\mathrm{d}} \neq 0$ modifies both the dynamics and the static properties of the $\mathrm{BH}$ model. An exhaustive analysis of possible phases has been published in [10, 11]. In our system the disorder is due to bichromaticity of the lattice, created by using two incommensurate laser pulses. This is not entirely equivalent to true random on-site potential as in [8]. Nevertheless for such a strong disorder, for not too big $J$ the system is in a gapless, insulating Bose glass phase.

Let us note that the spectrum of the autocorrelation function, Fig. 8, is indeed much denser than in the absence of disorder: this is easy to understand, as random variance of the local energy increases likelihood that sev-

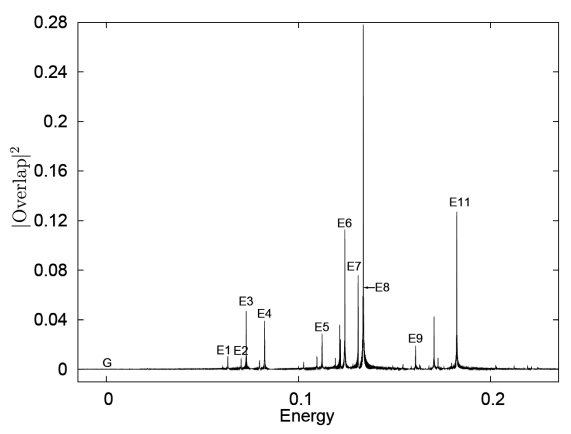

Fig. 8. Fourier transform of the autocorrelation function, Eq. (4), obtained dynamically for $s=14$ after switching on the lattice with the secondary lattice of strength $s_{\mathrm{d}}=2.1875$ (151 particles on 81 sites). Several peaks represent excitations populated during turning on of the lattices. The ground state is populated with less than $1 \mathrm{ppm}$ probability.

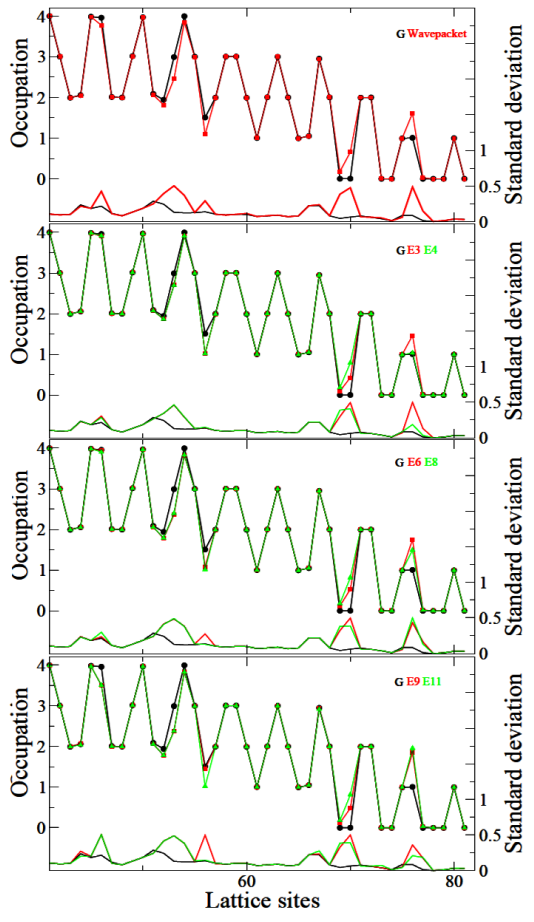

Fig. 9. The top graph compares the dynamical wave packet and the ground state, subsequent graphs compare the ground state (black on each plot) to the significantly populated excited states constituting the wave packet obtained for $s_{\mathrm{d}}=2.1875$. The right hand axis shows standard variation of the occupation number.

eral sites have similar on-site energies, providing opportunity for low energy excitations. For $J=0$, the analysis in terms of local energy cost for adding a new particle, developed in the absence of disorder, is still valid. The difficulty is that - because of the random fluctuations of the on-site energy - there is no way of classifying the sites as attached to a well defined plateau. This implies that a slightly different realization of the disorder 
will produce a different landscape of low energy excitations. Nevertheless, only a handful of states are significantly excited. In Fig. 9, the most significant excitations have been plotted. A noteworthy difference with the no disorder case is that, although excitations are local in nature, they are not single particle excitations. Each excited state and the ground state have similar occupation and particle number variance at all sites, except a few sites where the occupation is different, and particle number fluctuations are bigger.

The analysis performed in the previous section may also be done for the symmetric eigenstates. The results are analogous - peaks in the autocorrelation function graph split into several peaks corresponding to symmetry-broken eigenstates of the unsymmetric system.

\section{Conclusions}

We have described how the excited states may arise when evolving an ultracold gas in an optical potential - as a result of going through a superfluid-insulator quantum phase transition. In the absence of disorder, the excitations are local, one or a few particles are misplaced with respect to the ground state. The excitations appear only between edges of the Mott regions creating long range correlations.

The wave packet is a sum of states differing from the ground state by a single or a few particle elementary excitations and therefore similar in nature. Most notably in all presented states (in the Mott insulator region), all states have almost the same average occupation, very similar particle number variance. Still the wave packet is not a single eigenstate but a quantum superposition. This fact leads to the rise of nonlocal correlations throughout the sample. It may be directly observable as an increased variance of nonlocal observables such as number of particles in one half of the system. If one measured the number or particles to the left of the middle site, the statistical distribution stemming from independent realizations of the experiment would have a large number variance, much larger than the local particle number variance at the given site. This directly follows from the symmetry-breaking description and the presented data, as different eigenstates building the wave packet have different number of particles in one half of the system (jump of a particle from one half of the system to the other) than in the other half.

Slight symmetry breaking allows to analyze constituents of the symmetric eigenstates. It turns out that excitations differing by a long range particle jump are less probable, but not completely negligible.

A few questions arise. Firstly, we have considered only the deep lattice regime. If the lattice was shallower, what would be the nature of excitations present in the system? Secondly many more excitations would be present if the system was in the significantly nonzero temperature - how would it affect properties of the system such as the mentioned long range coherence and its decoherence?

\section{Acknowledgments}

We are grateful to K. Byczuk for pointing out the Gersch and Knollman paper [4]. Support within Polish Government scientific funds for 2009-2012 as a research project is acknowledged. M.Ł. is grateful for support within Jagiellonian University International Ph.D. Studies in Physics of Complex Systems (agreement No. MPD/ 2009/6) provided by Foundation for Polish Science and cofinanced by the European Regional Development Fund. Computer simulations were performed at ACK Cyfronet AGH as a part of the POIG PL-Grid project (M.Ł.) and at ICM UW under grant No. G29-10 (J.Z. and M.Ł.).

\section{References}

[1] D. Jaksch, C. Bruder, J.I. Cirac, C.W. Gardiner, P. Zoller, Phys. Rev. Lett. 81, 3108 (1998).

[2] M. Greiner, O. Mandel, T.W. Hänsch, I. Bloch, Nature 415, 39 (2002).

[3] J.D. Jackson, Am. J. Phys. 76, 8 (2008).

[4] H.A. Gersch, G.C. Knollman, Phys. Rev. 129, 959 (1963).

[5] Ph. Courteille, R.S. Freeland, D.J. Heinzen, Phys. Rev. Lett. 81, 69 (1998).

[6] F.K. Fatemi, K.M. Jones, P.D. Lett, Phys. Rev. Lett. 85, 4462 (2000).

[7] D.J. Papoular, G.V. Shlyapnikov, J. Dalibard, Phys. Rev. A 81, 041603 (2010).

[8] M.P.A. Fisher, P.B. Weichman, G. Grinstein, D.S. Fisher, Phys. Rev. B 40, 546 (1989).

[9] L. Fallani, J.E. Lye, V. Guarrera, C. Fort, M. Inguscio, Phys. Rev. Lett. 98, 130404 (2007).

[10] T. Roscilde, Phys. Rev. A 77, 063605 (2008).

[11] X. Deng, R. Citro, A. Minguzzi, E. Orignac, Phys. Rev. A 78, 013625 (2008).

[12] T.W.B. Kibble, J. Phys. A 9, 1387 (1976).

[13] W.H. Zurek, Nature 317, 505 (1985).

[14] J. Dziarmaga, A. Smerzi, W.H. Zurek, A.R. Bishop, Phys. Rev. Lett. 88, 167001 (2002).

[15] W.H. Zurek, U. Dorner, P. Zoller, Phys. Rev. Lett. 95, 105701 (2005).

[16] A. Polkovnikov, Phys. Rev. B 72, 161201 (2005).

[17] J. Zakrzewski, D. Delande, Phys. Rev. A 80, 013602 (2009).

[18] S. Ostlund, S. Rommer, Phys. Rev. Lett. 75, 3537 (1995).

[19] U. Schollwöck, Ann. Phys. 326, 96 (2011).

[20] G. Vidal, Phys. Rev. Lett. 91, 147902 (2003); ibid., 93, 040502 (2004).

[21] S.R. White, A.E. Feiguin, Phys. Rev. Lett. 93, 076401 (2004). 